\title{
Artificial Intelligence Based Body Sensor Network Framework-Narrative Review: Proposing an End-to-End Framework using Wearable Sensors, Real-Time Location
} Systems and Artificial Intelligence/Machine Learning Algorithms for Data Collection, Data Mining and Knowledge Discovery in Sports and Healthcare

Ashwin A. Phatak ${ }^{*}$ (D), Franz-Georg Wieland ${ }^{2}$, Kartik Vempala ${ }^{3}$, Frederik Volkmar ${ }^{1}$ and Daniel Memmert ${ }^{1}$

\begin{abstract}
With the rising amount of data in the sports and health sectors, a plethora of applications using big data mining have become possible. Multiple frameworks have been proposed to mine, store, preprocess, and analyze physiological vitals data using artificial intelligence and machine learning algorithms. Comparatively, less research has been done to collect potentially high volume, high-quality 'big data' in an organized, time-synchronized, and holistic manner to solve similar problems in multiple fields. Although a large number of data collection devices exist in the form of sensors. They are either highly specialized, univariate and fragmented in nature or exist in a lab setting. The current study aims to propose artificial intelligence-based body sensor network framework (AIBSNF), a framework for strategic use of body sensor networks (BSN), which combines with real-time location system (RTLS) and wearable biosensors to collect multivariate, low noise, and high-fidelity data. This facilitates gathering of time-synchronized location and physiological vitals data, which allows artificial intelligence and machine learning (Al/ML)-based time series analysis. The study gives a brief overview of wearable sensor technology, RTLS, and provides use cases of AI/ML algorithms in the field of sensor fusion. The study also elaborates sample scenarios using a specific sensor network consisting of pressure sensors (insoles), accelerometers, gyroscopes, ECG, EMG, and RTLS position detectors for particular applications in the field of health care and sports. The AIBSNF may provide a solid blueprint for conducting research and development, forming a smooth end-to-end pipeline from data collection using BSN, RTLS and final stage analytics based on Al/ML algorithms.
\end{abstract}

\footnotetext{
*Correspondence: a.phatak@dshs-koeln.de; ashwin.phatak@gmail.com

${ }^{1}$ Institute of Exercise Training and Sport Informatics, German Sports

University, Cologne, Germany

Full list of author information is available at the end of the article
} 
Keywords: Wireless body area networks, Wearable biosensors, Sports analysis, Real-time location system, Multisensor fusion, Vitals data

\section{Key Points}

- A large number of wearable sensor technologies have given rise to big data collection possibilities in the fields of sport and healthcare.

- Emergence of body sensor networks, real time location systems and multi sensor data fusion algorithm show great potential for application in wide set of industries.

- The proposed AIBSNF framework has potential to provide a solid blueprint for exploiting these rising technologies for end-to-end application from data collection to knowledge discovery across industries.

\section{Introduction}

\section{Big Data and the Future}

'Dataism' is a term coined by Yuval Harari in his popular science book 'Homo Deus'. This term suggests that in the near future, decisions in all aspects of society will be based on the interpretation of the available 'big data' [1]. "Big data is defined as high-volume, high-velocity, high-variety and high veracity information assets (4Vs) that demand cost-effective, innovative forms of information processing for enhanced insight and decision making." In this definition, volume refers to the magnitude or size of the data, variety refers to structural heterogeneity in the dataset, velocity refers to the rate at which data are generated and veracity refers to the truthfulness or reliability of the data [2-4]. 'big data' currently holds tremendous untapped potential, which has possible applications in a multitude of industries, including but not limited to health care, banking and finance, security, aviation, astronomy, agriculture, and sports [2, 5-7]. Although big data can be a considerable asset for the knowledge discovery process, using this data is a non-trivial task. Due to its unique computational and statistical challenges, the strength of 'big data' in terms of the $4 \mathrm{Vs}$ described above can also be its drawback. Noise accumulation, spurious correlation, measurement errors, and high computational power requirements are some of these challenges [8]. Solutions for any of the above-mentioned problems in terms of framework and data mining tools may prove crucial for generating data, analyzing it, and extracting actionable knowledge for application in the respective industries.

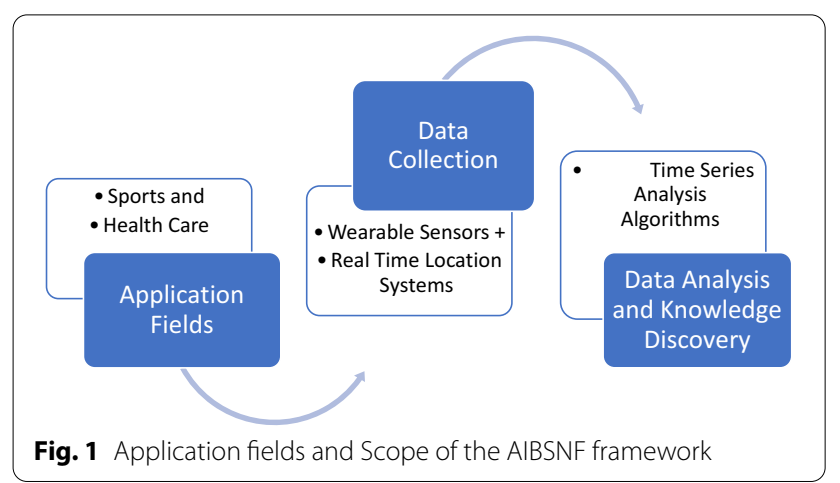

In recent years, the application of big data, its acquisition, and analysis using $\mathrm{AI} / \mathrm{ML}$ algorithms have been applied in sports and healthcare diagnostics [5]. It has resulted in improvements in the identification of critical information and is being used in decision-making processes [5]. The nature of these fields is such that certain physiological signs that signify sports performance are also good indicators of mental and physical health [5]. The physiological information and movement patterns required to investigate athletic performance in sports, such as heart activity, recovery, muscular strength coordination, balance, etc., have considerable overlap with general health indicators. Considering this overlap, the data collection tools for these physiological indices can potentially be used to analyze both sports performance and available health predictors [9].

Figure 1 outlines the scope of the present review, it outlines the field of application, technologies used for data collection and post data collection analysis for knowledge discovery in the broad set of fields.

\section{Status Quo of Data Gathering, Mining and Analytics in Sports}

The use of big data and AI/ML tools in sports was first introduced in track and field, and weightlifting [10]. Baseball was one of the first sports to use data for recruiting and performance-enhancing purposes [6]. The sports of basketball and football soon caught up with a large number of professional teams and academics using 'big data' for recruiting, performance analysis, and performance enhancement in their respective sports [7]. The data currently collected in sports falls under broad categories, viz. physiological data, position tracking data, psychological data, scouting data, 
video data, etc. [7]. Spatiotemporal events, position data, and comprehensive match statistics for several sports are commercially available today through companies such as OPTA (https://www.optasports.com/), Hudl (https://www.hudl.com/), Instat (https://insta tsport.com/), Statsbomb (https://statsbomb.com/), and others. Mining for the relevant information is primarily done through video and manual tagging [11]. The collection of physiological vitals such as muscular contraction data, GAIT analysis, etc., is currently difficult, if not impossible to extract just from the game footage. The quality of data available within the current technological limits is continuously improving, but some issues still persist in collecting high-quality data during live sporting events. Another issue is that data sources recorded by humans are currently prone to missing values, inconsistencies between different measures, and a temporal error [11]. Data sources that are automatically recorded, such as tracking information via motion capture systems, traditionally have difficulties with tracking complicated and crowded game situations [12]. Furthermore, video data's sheer size and complexity make it difficult for domain (sports) specific feature extraction [11]. This issue is sometimes dealt with manually or automatically after processing and cleaning the data; however, problems persist. Tracking a relatively small sporting object such as a ball traveling at high speeds is still unreliable even after post-event data mining [11]. Tracking high-speed objects such as balls and rackets is especially important since sporting equipment is usually the central reference point for meaningful sports analysis.

A recent review of sports research focusing on data mining and analytics (smart sports training) using AI/ ML algorithms showed that the most researched sports involving big data and computational intelligence were soccer, running, and weight lifting [2]. The same review identified a total of 97 studies ranging from individual to team sports with the same focus. It also concluded that there is a lot of room for improvement in research methods with respect to the quality and public availability of datasets, which provides opportunities to validate the research done. Furthermore, multiple studies have implemented or proposed frameworks of ML algorithms and artificial neural networks (ANN) for sports results prediction. They mainly focus on technical implementation of the algorithms and its performance while predicting outcomes of a sporting match [2]. There seems to be less emphasis on frameworks that focus on obtaining and organizing the high-quality, low noise, and time-synced data required for implementing these algorithms [13]. The framework proposed in the current review aims to address these points.

\section{Data Gathering, Mining and Analytics in Health Care}

The field of health care has a long history of recording, analyzing, and drawing inferences based on data [8]. This seems to be an effect of the requirements of regulatory bodies [8]. The total amount of healthcare data is predicted to cross Yottabyte scale in the next years [5]. Multiple approaches using AI have been used in the past for injury risk assessment and performance prediction [9]. Vison-based motion analysis has also been used for medical diagnostics[14]. There still seems to be untapped potential for big data to improve clinical operations, public health, preventive medicine, precision medicine, evidence-based medicine, remote monitoring, patient profiling, etc., in terms of lower cost, faster analysis, and reduced error rates [15]. A few of the factors crucial for accelerating innovation in the field of smart medicine seem to be, data gathering techniques and data mining from existing sources [16].

Architectural frameworks facilitating AI/ML have been proposed for analyzing the currently available vitals data across various sectors within healthcare [15]. Despite this, deep and smooth data integration across multiple healthcare applications is fragmented and slow. Another technical challenge for the development of such tools is the data from different healthcare environments. The lack of consistency in the structure of the data, available features, noise, and bias of the source may lead to issues in the trained algorithms [17]. With the development and advancements in low-cost near gold standard sensor technology, there is a possibility to combine multiple sensors to collect organized, time-synchronized data as the first step for developing a pipeline for use in numerous healthcare and diagnostics applications in the form of fusion technologies [18, 19]. Wearable sensors seem to be the ideal tool for collecting such high-quality data [20-22].

\section{Wearable Biosensors}

The rise of wearable sensors as tools for data collection seems to be ideal for gathering physiological and vital data. Hence, wearable sensors have become popular in medical, entertainment, security, and commercial areas [21]. A recent review published in 'Nature Biotechnology' elaborated the rising interest in wearable biosensor technology in academics, performance, and the health industry [22]. Wearables show great potential to provide continuous, real-time physiological data using dynamic non-invasive measurements of biochemical and physiological markers. So far, these sensors have been used for gathering precise, high-fidelity strategic data, which facilitate a whole host of applications with, military, precision medicine, and the fitness industry at its forefront [23-25]. Their precise fidelity 
Table 1 A non-exhaustive list of sensors and their applications in physiological vitals data gathering

\begin{tabular}{|c|c|c|c|c|}
\hline Category & Physiological index & Application & Types of sensors & References \\
\hline \multirow[t]{2}{*}{ Sweat monitoring } & Glucose and lactate & $\begin{array}{l}\text { Blood sugar and physiological } \\
\text { load monitoring }\end{array}$ & $\begin{array}{l}\text { lontophoresis electrode-based } \\
\text { sensors }\end{array}$ & [20] \\
\hline & Electrolyte concentration & $\begin{array}{l}\text { Hydration monitoring, measuring } \\
\text { of trace mineral densities, etc. }\end{array}$ & Galvanic skin resistance (GSR) & \\
\hline \multirow[t]{2}{*}{ Temperature sensors } & $\begin{array}{l}\text { Skin/core temperature measure- } \\
\text { ment }\end{array}$ & Acute infection monitoring & Pyroelectric sensors & {$[30]$} \\
\hline & Cortisol & Stress monitoring & $\begin{array}{l}\text { Molybdenum disulfide } \\
\text { nanosheets }\end{array}$ & {$[31]$} \\
\hline Emotion regulation & Skin conduction & Continuous estimation of stress & GSR & [32] \\
\hline \multirow[t]{3}{*}{ Respiration } & Oxygen saturation & Blood oxygen levels & Light emitting diode (LED) & {$[23,33]$} \\
\hline & Respiratory effort sensor & & Strain sensor & \\
\hline & Stretchable respiration sensors & $\begin{array}{l}\text { Respiratory disease monitoring } \\
\text { and diagnosis }\end{array}$ & Resistive humidity sensors & [28] \\
\hline \multirow[t]{2}{*}{ Cardiological data } & Heart rate & Physical load & Electrocardiogram (ECG) & {$[34]$} \\
\hline & Heart rate variability & Recovery and stress & & \\
\hline \multirow[t]{2}{*}{ Skeletal muscle analysis } & Electrical activity in muscles & $\begin{array}{l}\text { Muscular abnormities, nervous } \\
\text { system functioning }\end{array}$ & Electromyogram (EMG) & [35] \\
\hline & & $\begin{array}{l}\text { Muscular fatigue, injury prob- } \\
\text { ability }\end{array}$ & & \\
\hline \multirow[t]{2}{*}{$\begin{array}{l}\text { Continuous exercise monitor- } \\
\text { ing through body motion } \\
\text { analysis }\end{array}$} & Biomechanical data & $\begin{array}{l}\text { Body position, postural control } \\
\text { and team interaction }\end{array}$ & $\begin{array}{l}\text { Accelerometers, pressure sen- } \\
\text { sors, inertial measurement unit } \\
\text { gyroscope }\end{array}$ & {$[36,37]$} \\
\hline & Foot movement pattern & GAIT analysis & & \\
\hline
\end{tabular}

and precision varies based on the specifications of their varying use cases.

Advances in electronics, printing, non-invasive data collection, and monitoring technology, have given rise to durable, unobtrusive, non-invasive wearable clothing as an electronics platform capable of sensing human locomotion and vital physiological signals [21, 25-29]. Such media and miniaturization of sensors provide unprecedented capacity to gather a wide range of data in many scenarios. By choosing a specific set of sensors strategically located at different human body locations, there is potential to collect precise data for solving interesting problems. Table 1 shows a non-exhaustive list of noninvasive sensor technology that can potentially be used in various combinations to gather physiological data for applications in a wide range of disciplines.

\section{Real-time Location Systems (RTLS) in Healthcare and Sports}

Real-time location system (RTLS) is a combination of wireless hardware and software deployed to acquire a continuous real-time position of assets and resources, usually using a fixed reference point or receivers [38]. They seem to have an advantage over video capture as in most situations direct line of sight is required for motion analysis and this may not always be possible [39]. Most RTLS technologies are capable of measuring ToA (time of arrival), TDoA (time difference of arrival), AOA (angle of arrival), RSS (receiver signal strength), RSF (receiver signal phase), and RTF (roundtrip time of flight). Using this, they can identify the real-time location of an object(s) in question with one of the following methods: literation, angulation, or fingerprinting [40]. In recent years due to advancements in data collection technology, improvements in data mining algorithms and a reduction in the cost of development kits have given rise to a whole host of applications in industries such as production management, food delivery, healthcare and sports analytics [38, 41-44].

In healthcare, RTLS has been used effectively in elder care tracking, medical asset tracking, medication tracking, etc. Radio-frequency identification (RFID) has mainly been used in various capacities for improving medical asset management [34]. Furthermore, due to the COVID-19 pandemic of early 2020, RTLS was used for contact tracing to identify the potential spread of the virus. A combination of RTLS and electronic medical records was successfully able to locate all contacts with a sensitivity of $77.8 \%$ and specificity of $73.4 \%$. Although not perfect, there seems to be potential to improve this rate by integrating other complementary measurement techniques [45].

In the field of team sports, real-time position and event data, in particular, has become crucial for the industry. 
Table 2 A non-exhaustive list of real-time location systems (RTLS) and applications thus far along with their specifications. (NLoS=no line of sight, LOS = line of sight,

\begin{tabular}{|c|c|c|c|c|c|}
\hline Technology & Application & Dynamic accuracy & Detection range & $\begin{array}{l}\text { Transmission } \\
\text { frequency range }\end{array}$ & References \\
\hline mmWave (5 g) & Human Pose detection & Up to $<0.02$ m @ LoS & >200 m@ outdoor & $30-300 \mathrm{GHz}$ & {$[47,48]$} \\
\hline $\begin{array}{l}\text { Active radio-frequency } \\
\text { identification (RFID) }\end{array}$ & $\begin{array}{l}\text { Indoor location detection } \\
\text { of devices and people }\end{array}$ & 0.9 to 1.6 m@ NLoS & $\begin{array}{l}\text { 50-95 m radius with pos- } \\
\text { sible scalability to } 1000 \text { m } \\
\text { @ outdoors }\end{array}$ & 433 & {$[42]$} \\
\hline $\begin{array}{l}\text { 3D-Light Detection and } \\
\text { Ranging (LiDAR) }\end{array}$ & $\begin{array}{l}\text { Precision vehicle localiza- } \\
\text { tion }\end{array}$ & 0.01 to $0.2 \mathrm{~m} @ \mathrm{LoS}$ & $\begin{array}{l}\sim 200 \mathrm{~m} @ \text { @oS, both } \\
\text { outdoor and indoor }\end{array}$ & 200 Thz & [49] \\
\hline Wireless Fidelity (Wi-Fi) & $\begin{array}{l}\text { Indoor and outdoor posi- } \\
\text { tioning for smartphones }\end{array}$ & 1-3 m @NLoS & $\begin{array}{l}<200 \text {, @ outdoor } \\
\text { and }<60 \text { m indoor under } \\
\text { Wi-Fi covered distance }\end{array}$ & 2.4 to $5 \mathrm{GHz}$ & {$[42]$} \\
\hline Ultra Sound & Indoor location & $\begin{array}{l}\text { Up to } 0.01 \mathrm{~m} @ \text { LoS } \\
\text { and 0.02@ NLoS }\end{array}$ & Up to10 m @LoS indoor & $1-20 \mathrm{MHz}$ & {$[46,50]$} \\
\hline Bluetooth & $\begin{array}{l}\text { Real-Time Indoor Posi- } \\
\text { tioning }\end{array}$ & $\begin{array}{l}\text { Typically, between } 2 \text { and } \\
5 \mathrm{~m} \text { but can go up to } \\
0.77 \mathrm{~m} \text { using different sig- } \\
\text { nal processing algorithms }\end{array}$ & Up to 2 m @ NLoS & $\begin{array}{l}2 \mathrm{MHz} \text { of width in the } \\
2.4 \mathrm{GHz} \text { band }\end{array}$ & {$[40,51]$} \\
\hline Ultra-Wide Band (UWB) & $\begin{array}{l}\text { Tracking and position } \\
\text { detection in sports }\end{array}$ & $\begin{array}{l}\text { Between } 0.08 \text { and } 0.2 \text { m } \\
\text { @ LoS }\end{array}$ & $40-80 \mathrm{~m}$ & 3.1 to $10.6 \mathrm{GHz}$ & {$[42,46,52]$} \\
\hline \multirow[t]{2}{*}{ Computer Vision } & $\begin{array}{l}\text { Tracking of ball in sports } \\
\text { such as Tennis and Cricket }\end{array}$ & $\begin{array}{l}\text { Up to 0.05.-0.1 m @ } 340 \\
\text { fps }\end{array}$ & N/A & N/A & {$[40,53]$} \\
\hline & $\begin{array}{l}\text { Tracking path length of } \\
\text { multiple objects }\end{array}$ & $\begin{array}{l}\text { Up to } 8.5 \% \text { error and } \\
\text { under } 1 \mathrm{~m} \text { for marker- } \\
\text { based solutions }\end{array}$ & N/A & N/A & \\
\hline $\begin{array}{l}\text { Global Positioning System } \\
\text { (GPS) }\end{array}$ & $\begin{array}{l}\text { Measuring real-time } \\
\text { movement of soccer play- } \\
\text { ers in a test situation }\end{array}$ & $\begin{array}{l}\text { Up to } 1.31 \mathrm{~m} / \mathrm{s} \text { error while } \\
\text { measuring velocity and } \\
6.05 \% \text { error when meas- } \\
\text { uring position @ NLoS }\end{array}$ & $\begin{array}{l}>100 \mathrm{~km} \text { Outdoor and } \\
\text { Indoor }\end{array}$ & $\begin{array}{l}1575.42 \mathrm{MHz} \text { and } \\
1227.6 \mathrm{MHz}\end{array}$ & {$[54]$} \\
\hline $\begin{array}{l}\text { Global Navigation Satel- } \\
\text { lite System }\end{array}$ & Smartphone Location & $\begin{array}{l}\text { Up to a few centimeters } \\
\text { but unstable }\end{array}$ & $>100$ km Outdoor & $1-2 \mathrm{GHz}$ & {$[55]$} \\
\hline
\end{tabular}

Findings from this gathered data have benefited the field for physiological indicator analysis, tactical analysis, and their combination [46]. Despite considerable progress in motion analysis systems, there seems to be a lack of accurate and cost-effective technologies in the current market. The accuracy levels required for different marker-less human motion analysis scenarios are yet not established, but can potentially be improved by adding wearable tags (transmission antennas) on the players/sporting objects [46]. Multiple technology stacks and frameworks for data storage and potential analysis using $\mathrm{AI}$ and ML algorithms have been proposed [3]. Albeit, the gathering of such data in a synchronized manner is a non-trivial task. Multiple companies offer position data gathering and interpretation services using real-time location systems (RTLS) technology, but studies on the accuracy of this data are limited. Table 2 shows a non-exhaustive list of contemporary RTLS technologies, their accuracy, and detection range, in different applications.

The fields of healthcare and sports have, to date, used RTLS and wearable technologies separately for solving specific problems $[43,45,54]$. The scope of the application has been limited thus far, but there seems to be a massive potential for using RTLS in combination with wearable sensor technology. Previous research has proposed and implemented frameworks in health care and sports using these technologies separately. Still, there seems to be a lack of integration of these two technologies.

\section{Body Sensor Network (BSN)}

Developments in wearable sensor technology and the improvements in wired and wireless communication devices have given rise to low-power, intelligent, miniaturized sensor node networks, also known as wireless body areas networks, body sensor network, body area networks, etc. (referred to as BSN henceforth) [56]. The BSNs provide a blueprint for placing sensors at strategic locations for each individualized application. BSNs have been proposed in a multi-level fusion framework (MLFF) to monitor soldiers and help decision-making by using multiple factors such as physiology, emotions, fatigue, environment, and location. The same MLFFs, which can potentially measure soldiers' performance, can be used for sports performance analysis and health care with minor tweaks [24]. 
Biomechanical, biometric, and positional data are crucial for understanding physiology and logistics involved in sports. The data thus obtained, both in real-time and post-event, has tremendous potential in knowledge discovery, research and development across sports. This combination may help decode physiology and logistics in a sport, which may unlock further avenues for research and development in academics or applications in industry. Currently, several AI/ML algorithms exist which have the potential for performing multi-sensor data fusion. These algorithms can stitch images, perform time series analysis and forecasting, automatic event detection and classification, anomaly detection, fault detection, etc. [46-48].

\section{Artificial Intelligence and Machine Learning Algorithms for Multi-sensor Data Analysis}

A comprehensive range of tools and techniques for time series analysis already exist for multidimensional signal processing. The utility has been demonstrated in applied and fundamental research in physics, biology, medicine, and economics [57]. A growing number of time series analysis algorithms have become available as data mining and interpretation tools due to recent advances in AI and ML. They seem to have an excellent tool capable of handling multivariate data. Table 3 shows a non-exhaustive list of algorithms used for multiple analogous applications that have the potential to be used in the problems addressed by the current review.

A large number of algorithms are continuously being developed in the field of AI/ML-based time series analysis. Multiple libraries in Python and $\mathrm{R}$ exist along with open-source repositories on GitHub. There are various tools available for analyzing and interpreting multivariate data acquired from multiple sensors $[18,19,58]$. However, multiple sensors in different fields seem to be univariate or exist only as theoretical frameworks. There appears to be limited applications of using continuous, time-synchronized physiological vitals and RTLS data in combination with AI/ML algorithms both, in sports and healthcare.

The present review aims to highlight available tools in fields of wearable biosensors, RTLS, and AI/ML. Furthermore, the authors propose, AIBSNF, a framework of BSN which collects continuous multivariate physiological and live location data through the mashup of RTLS and wearable sensor technology in a potentially time synchronized manner. AIBSNF provides the blueprint for collecting such data, which is ideal for knowledge discovery through AI/ML algorithms. The authors of the current review highlight the framework's application in two widely distinct scenarios, viz. team sports (tackle in football) and healthcare diagnostics (monitoring and research in patients with rheumatoid arthritis or osteoarthritis).

\section{Artificial Intelligence-based Body Sensor Network Framework: AIBSNF}

The selection of the sensors and placement on the body is crucial due to the limitations on the number of sensors placed on a single person. Furthermore, the sampling rate for different sensors may be different, which needs to be considered depending on the applications at hand. In Table 4, we suggest an example BSN framework and strategic placement of selected sensors based on successful past applications and fit into the conceptual framework shown in Fig. 2.

Figure 3 illustrates the whole proposed framework by using sample sensors, their ideal placement, data gathering and preprocessing as synchronizing the data, use of time series analysis algorithms for specific used case of sport specific event detection.

\section{Specific Applications}

\section{Monitoring and Research in Patients with Rheumatoid Arthritis or Osteoarthritis}

Rheumatoid arthritis (RA) is a chronic inflammatory autoimmune disorder that affects the joints but can also cause damage to other systems such as skin and lungs. It is projected that over 78 million adults will be diagnosed with rheumatoid arthritis in the US alone. Among those with arthritis, one in four have movement and working limitations. Furthermore, adults with arthritis were shown to be 2.5 times likely to have fallen as compared to healthy adults (https://www.cdc.gov/arthritis/data_stati stics/arthritis-related).

Multiple studies have explored compliance rates as compared to the gold standard while performing rehabilitation exercises in patients with knee osteoarthritis. This was done using IMU sensors for feedback and monitoring resulting in varying degrees of accuracy during measurements. The studies concluded that wearable technology for assessing rehabilitation performance is a viable solution with room for potential improvements in measurement accuracy and compliance with the gold standard $[75,76]$. Furthermore, ECG abnormalities have been detected in patients with RA, and there is a need to explore this area further [70].

AIBSNF can be used to continuously monitor individuals with arthritis. According to previous research there are observed reductions in stride length, EMG activation of specific muscles, and abnormalities in the ECG pattern (all known markers of progression of arthritis) [70, 71]. Interventions could be potentially timed to manage attrition in RA patients using medical or nonmedical information, such as exercise prescription and change in medications. Furthermore, the data collected from such 


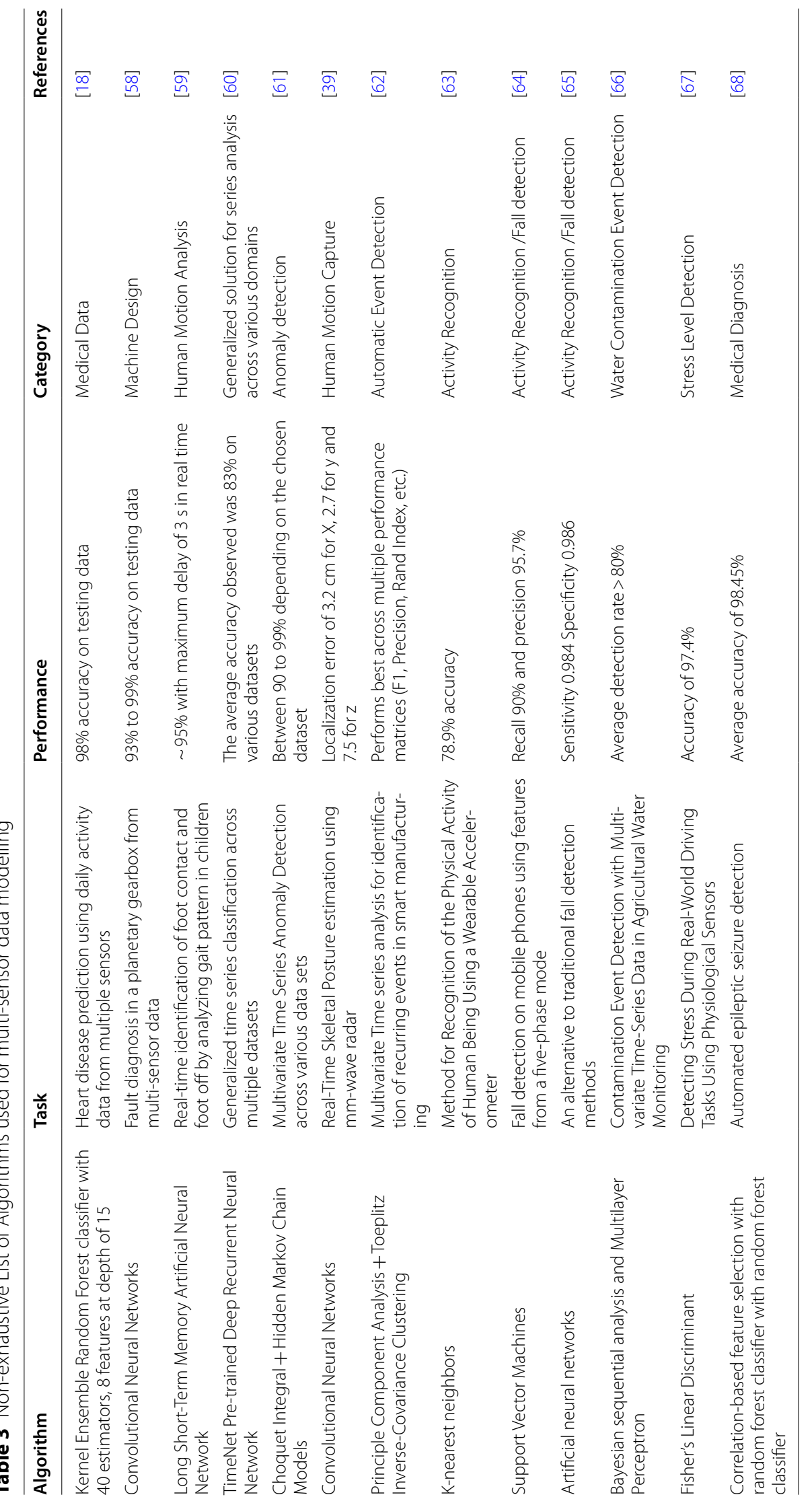




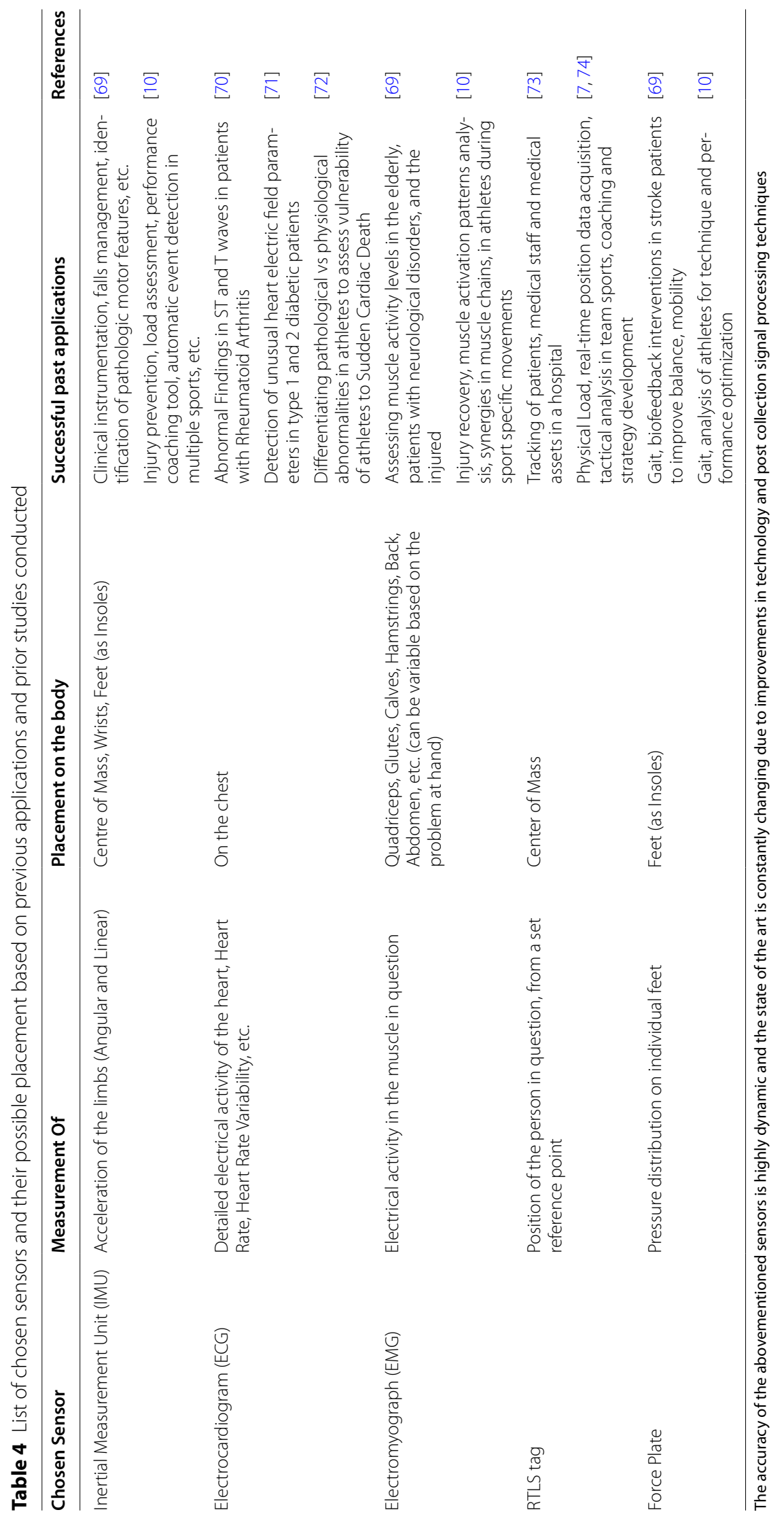




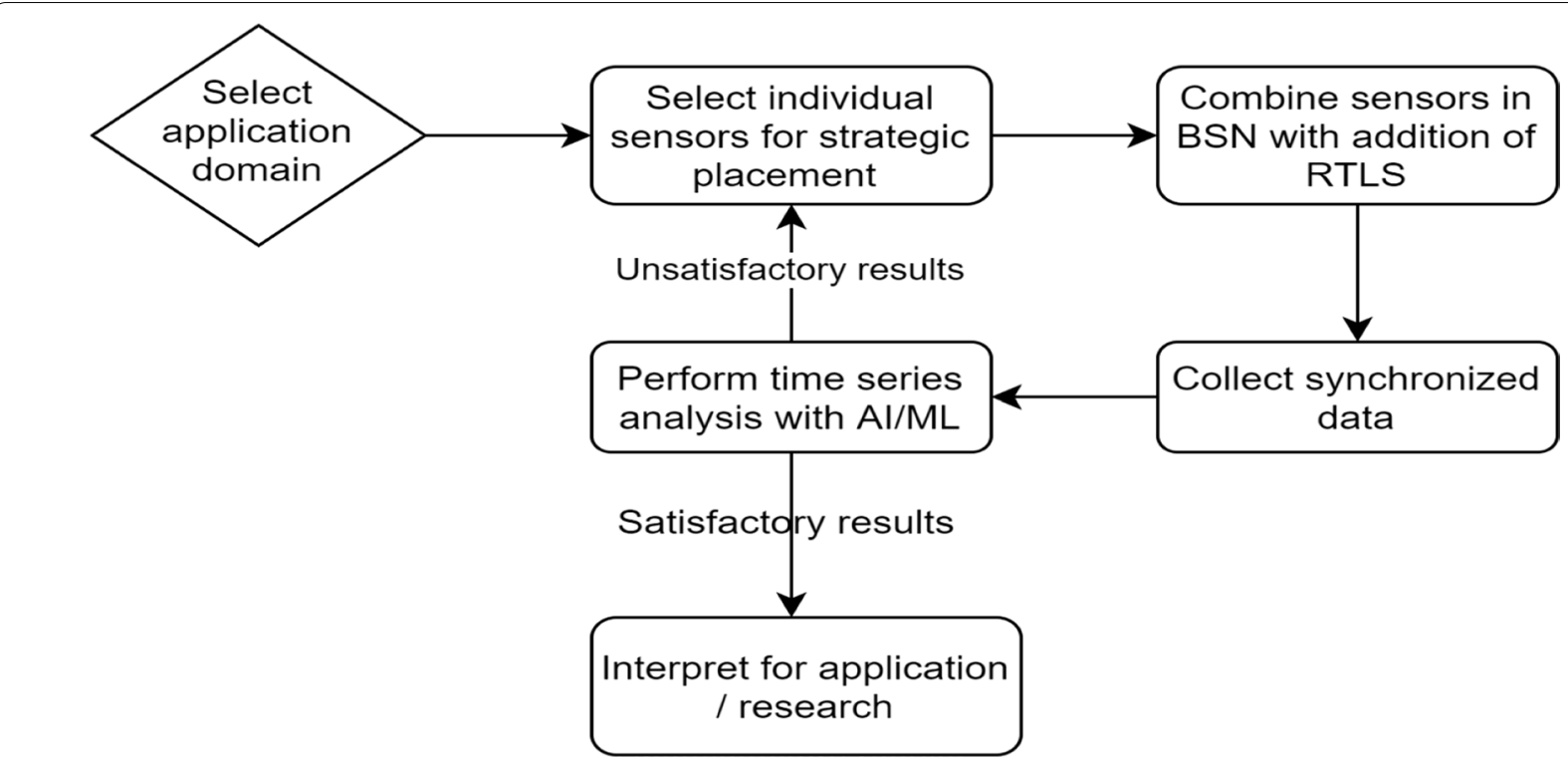

Fig. 2 The conceptual framework (AIBSNF) for building a knowledge discovery pipeline

continuous monitoring can be used by doctors, researchers and other health professionals to measure the efficacy and compliance rates of the intervention.

\section{Example: Automatic Detection of a Tackle in the Game of Football}

With an increasing number of teams taking decisions based on data analytics, there is a requirement for gathering and interpreting many high-fidelity data in sports specific scenarios. Automatic event detection and accurate position data are both crucial for this purpose. Figure 4 outlines a procedure in which ECG, EMG, gyroscopes, and RTLS tags have been placed at strategic locations on players from opposing teams and the ball. All these sensors would collect real-time data which convey the biomechanical and vital information from players, as well as the ball's position. The scenario in Fig. 4 is a tackle which has a unique visual fingerprint, i.e., where the player without the ball (player 2) is on the ground with one leg extended, trying to reach the ball. Player 1 is trying to dribble trying to reach the ball, which is in the possession of player 1 .

Human experts can identify a tackle when they see it. This is primarily due to the physical interaction of two players, which is unique to the tackle itself. When this information is digitized using the BSN, the physics of the ball and biomechanics, combined with the location data of all three parties involved can be recorded. This data can be used for automatic sports-specific event and lower limb movement detection [77, 78]. A time-series clustering and classification algorithm can potentially identify all sets of tackles automatically. Another advantage of tracking ECG, EMG, and RTLS data is tracking of physical load on the cardiovascular system and individual muscles can also be performed. When done on an ongoing basis, there is potential to avoid injury, assess the preparedness of an athlete, and find new correlations in a host of technical and tactical components of the game in real-time $[7,74]$.

The same methodology can potentially be applied across multiple individual and team sports to identify a wide variety of events. Automatic event identification has been proposed in previous studies, but there is further research warranted due to low reliability and validity of existing approaches [37, 77]. The current BSN plus time series analysis framework would potentially prove invaluable for multiple applications in sports, including but not limited to analyzing technique, coaching, self and opponent analysis, tactical analysis, talent identification, player selection, recruitment etc. [7, 74, 79]. Furthermore, broadcasting agencies can use such data to provide visualizations and real-time information breakdown for live sporting events. This may help enrich the ordinary viewer's experience, providing them with in-depth information from an expert's perspective. Health performance tracking is another up and coming field due to the rise of high-quality low-cost sensors. AIBSNF can be potentially used to build biofeedback mechanisms for continuous health tracking for a whole host of applications such as sleep and recovery tracking, personalized training programs for strength, mobility, cardiovascular endurance, and even ergonomic posture feedback in workplaces [56]. 


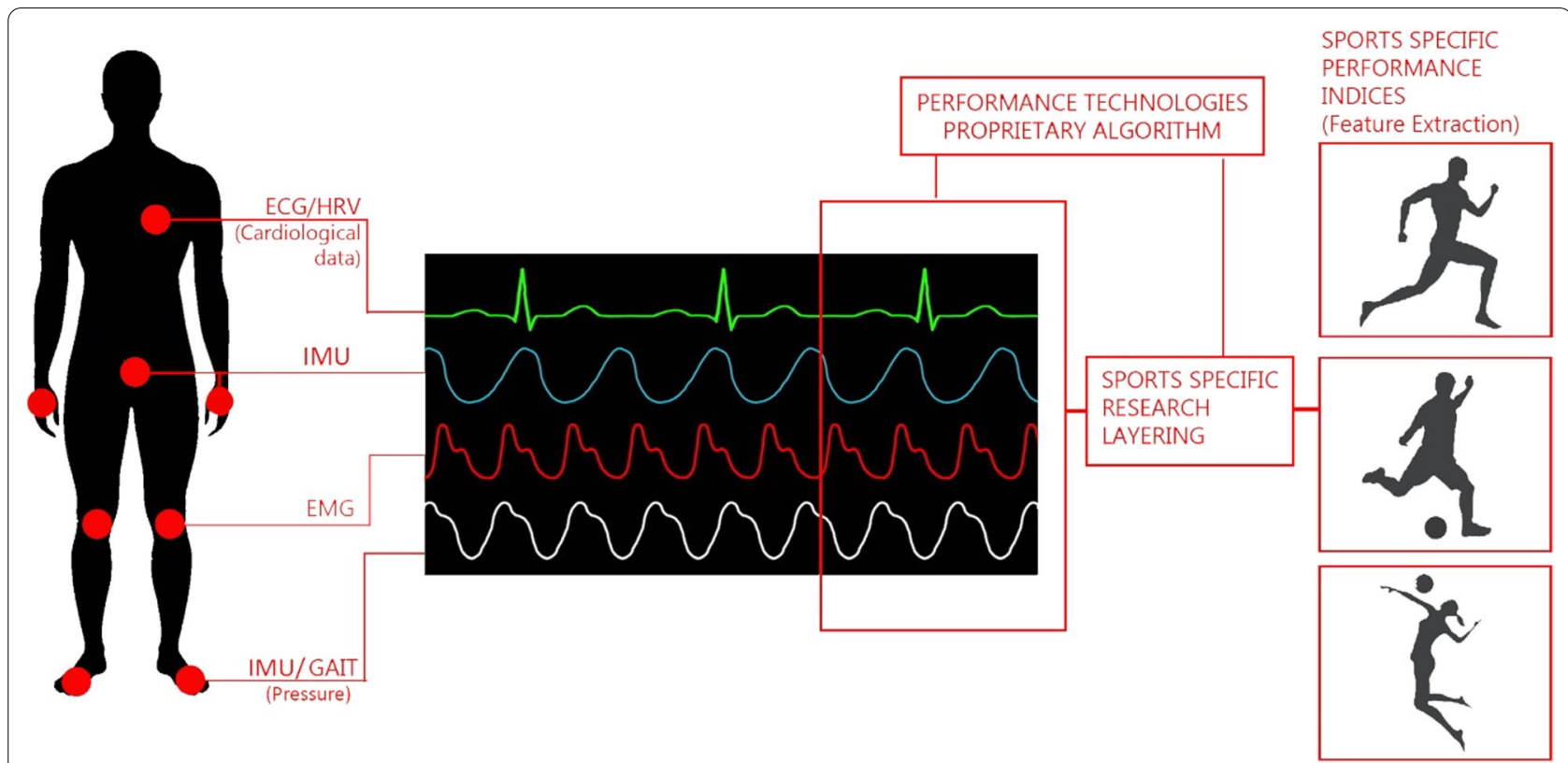

Fig. 3 Example framework with selected BSN for continuous monitoring of chronic diseases and sports specific event detection

\section{General Applications}

\section{Smartphone Applications and Wearables in Fitness and Health Tracking}

Due to properties such as utility, portability, high computing power, the smartphone has become an ideal tool for collecting a wide variety of data including health-related parameters in everyday life [64]. Using technologies such as WiFi and bluetooth, these devices provide a perfect ground to stack systems of data transmission from a wide range of sensors in a simplified manner. The collection of data can be achieved by the smartphone itself with embedded sensors (e.g., triaxial accelerometer) or with the use of external sensors (e.g., ECG, EMG) using wearables [80]. Furthermore, the smartphone can potentially act as a data transmitting and processing tool that collects data and interacts with it. These properties facilitating input, output and interactive operations make the smartphone an important part of the data management systems [21]. Various smartphone applications combine data transmitted from different sensors which can be analyzed both in real-time and post collection [81].

Mashup data collection tools have been applied in several studies such as the "Physiodroid study" [82]. Using external sensors and a smartphone for collection and computation, the team simultaneously analyzed ECG, heart rate, respiration, acceleration and skin temperature. Data was transmitted into an app where the patients and the clinicians had insights into the processed data. Such data can prove useful to detect emergency events and have shown to reduce anxiety in patients [82].

Besides the medical sector, ordinary users can profit from such integrated systems when applied in individual sports. Connecting data on e.g., muscle oxygen levels with heart rate there is potential for having high fidelity view on the condition of the body during training sessions, which could be useful for managing physical load and intensity on a personalized level.

\section{Applications in Healthcare Diagnostics and Research}

A set of clinical case studies performed with patients on the autism spectrum compared the use of multivariate and univariate data using ML methods, to demonstrate the utility of multivariate analysis. The authors concluded that multivariate analysis techniques seem crucial for analyzing data collected from biological networks [83]. Furthermore, most diseases seem to have multiple causes and prognosis which are usually determined by several factors. The multivariable analysis allows accounting for the multifaceted nature of risk, and their relative contribution to the result. Hence it seems to be advantageous to gather multivariable data for diagnosis and designing probable interventions [84]. In line with previous research the proposed AIBSNF framework can be integrated in textile-based wearables [29]. These can potentially be self-powered, e.g. using recent advances in piezoelectric technology by harvesting energy from biomechanical movement $[27,85]$. The wearables can also 


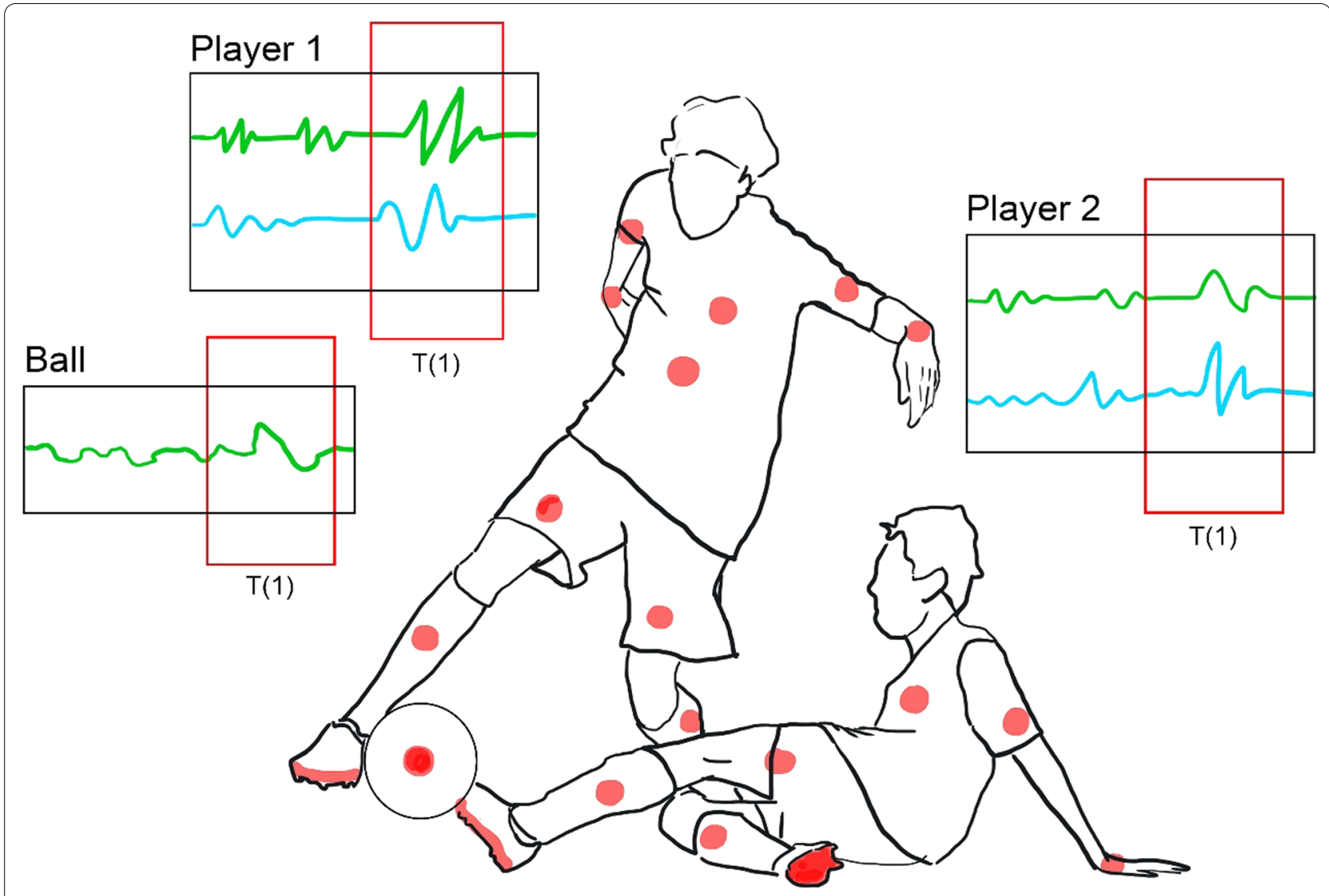

\begin{tabular}{|l|l|l|}
\hline \multirow{2}{*}{ Player 1 } & Sensor 1 & \\
\cline { 2 - 3 } & Sensor $\mathrm{n}$ & \\
\hline \multirow{2}{*}{ Player 2 } & Sensor 1 & \\
\cline { 2 - 3 } & Sensor $\mathrm{n}$ & \\
\hline Ball & IMU & \\
\hline
\end{tabular}

$T(1)$

Fig. 4 The scenario of conducting a tackle in the sport of football where both the players are fitted with a body sensor network

be extended to smart prosthetics and other assistive technologies [86].

\section{Applications in Alternative Medicine}

'Pulse diagnosis' is a diagnostic technique used in Ayurveda, traditional chinese medicine (TCM), and other alternative therapies. Practitioners of these alternative medicine fields understand the pathological changes in internal organs $[87,88]$. Devices capable of quantifying pulse via multi-sensor information using ECG, ultrasound imagining, pressure impedance blood flow, and volume pulse have been developed [89]. Furthermore, 
pulse diagnosis has also been conducted by measuring skin impedance at acupoints using a photoplethysmography sensor, galvanic skin response (GSR), and a smartphone. This was done to diagnose a condition called wiry pulse in TCM with an accuracy of above 90 percent [90]. Research in areas that combine modern technologies to quantify and validate ancient medicine practices, seem to require multivariate vitals data and its analysis. AIBSNF with slight modifications may prove valuable for conducting validation studies and develop alternative medicine technology.

\section{Playing Field for Artificial Intelligence and Machine Learning}

Chess has been used as an ideal scenario to push $\mathrm{AI} / \mathrm{ML}$ research forward. This is because each piece has a strict set of rules as to where it may move. Furthermore, performance in chess is highly objective due to the strict set of rules. This allows the digitization of all moves performed, which can then be studied using probabilistic mathematical modeling. This makes it ideal for training agents via reinforcement learning [91]. Using the proposed BSN and RTLS technology, real world sports can be digitized in the same manner. However, the players in sports don't follow rules as strict as those in chess. Sports are still largely contested under set of rules which are liberal as compared to chess. This, when digitized, can potentially provide a vast playground for $\mathrm{AI} / \mathrm{ML}$ algorithms. This may offer a multitude of insights into specific sports and may also help move the field of reinforcement learning forward.

\section{Limitations and Issues}

Although there is enormous potential for the use of BSNs, several challenges still exist for its implementation. On the technology side, the wearable sensors developed are comparatively new and collect data at different frequencies. They also lack benchmarking and approval for use in the medical industry [21]. The energy required for various sensors is varied, and there exist limitations such as data collection time due to the size and the shape of the battery, compliance of the user, and the physical impact of sensor operations. Hence, designing effective BSNs for solving problems requires a comprehensive set of domain-specific and technological knowledge [92]. Furthermore, on the analytics side, there still exist challenges such as computational power availability, high dimensionality, noise, and variable latency from different sensors in the case of real-time data collection [21]. The field of AI/ML, although advanced, is still in its infancy for discovering causal relationships. Hence, care must be taken to interpret output from AIBSNF as causal inferences or diagnosis, in the case of healthcare applications.

Data security is another major issue in the development and use of AIBSNF. Due to the intimate nature of the collected data, there needs to be mechanisms ensuring strict data protection at all proposed pipeline levels [56, 92]. The BSN connected to a user and data storage location may be significantly threatened by outside attacks. Data security risks may be higher for attacks from some entity with inside access to this data [22]. These issues are not unique to the BSN framework but for big data in general. However, due to the nature of the data collected in the applications mentioned in the current review, the threat may be amplified in terms of confidentiality, integrity, and information privacy [93].

In combination with data, using AI algorithms as independent decision making tools is a matter of great debate in the healthcare sector due to privacy concerns [94]. In the context of healthcare, AI can be categorized in two different ways, the first being a diagnostic tool, where the decision making and ethics lie with the human user of the tool. The second category is AI algorithms as independent decision makers or non-biological healthcare professionals [94]. The scope of the current paper lies within the prior category. A recent review outlines the opportunities and drawbacks of using $\mathrm{AI}$ in personalized medicine. It concludes the necessity for a multidisciplinary, public discussion to define the principles, ethics and social guidelines for using AI in healthcare due to limited expertise of the regulation sector in the field of concern [95].

\section{Conclusion}

The fields of RTLS and wearable biosensors are rapidly developing. There has been tremendous progress in improving accuracy, validity, and reliability in the sport and healthcare industries over the past decade $[22,40]$. It is safe to assume that they will continue to improve with the further layering of AI/ML techniques. AIBSNF seems to be ideally positioned to take advantage of the improvements in all these fields. It has the potential to impact a wide range of research and development activities in multiple industries. Due to the rapid pace of this development, numerous technological challenges exist. Identifying the right sensors, and mashing them up successfully at appropriate sample rates for time-synchronous data gathering, is possible but challenging. Data protection at each level of collection, use of the right algorithms, availability of computational power, and data science expertise are needed to successfully implement such technology on a commercial scale. The fields of sports and healthcare seem to be ideal areas where the proposed mashup technologies can be of significant benefit. AIBSNF provides 
a high-level understanding of how these technologies can be combined to develop applications in multiple fields. However, there still exist a whole host of technical challenges specific to the application domain. Further research and development are required for the successful application of AIBSNF in the highlighted industries.

\section{Abbreviations}

BSN: Body sensor networks; AIBSNF: Artificial intelligence-based body sensor network framework; RTLS: Real-time location systems; AI/ML: Artificial intelligence and machine learning; ECG: Electrocardiogram; EMG: Electromyogram; ANN: Artificial neural networks; GSR: Galvanic skin resistance; LED: Light emitting diode; IMU: Inertial measurement units; LoS: Line of sight; NLoS: No line of sight; MLFF: Multi-level fusion framework; RA: Rheumatoid arthritis; TCM: Traditional Chinese medicine.

\section{Acknowledgements}

The authors of the paper would like to acknowledge Ms Maithili Phatak for her contribution for the artwork in the current paper. The authors would also like to acknowledge the contributions of the management staff and language correction team of the Institute of Exercise Training and Sport Informatics at the German Sports University, Cologne.

\section{Authors' contributions}

AP: Primary author, writing and overall research, F-GW: Writing and reviewing of the scenarios and the proposed framework, KV: Researching and writing time series algorithms section, FV: Discussion and conclusion writing, DM: Supervisor, contributions in introduction, sports applications and framework development. All authors read and approved the final manuscript.

\section{Declarations}

\section{Funding}

Open Access funding enabled and organized by Projekt DEAL. No external funding was obtained for the purpose of the study.

\section{Availability of Data and Materials}

Materials required for the current review were previously published peer review articles which have been cited in the text and references.

\section{Ethics Approval}

Not applicable.

\section{Consent for Publication}

Not applicable.

\section{Competing interests}

Ashwin Phatak, Franz-Georg Wieland, Kartik Vempala, Frederik Volkmar and Daniel Memmert declare that they have no conflicts of interest relevant to the content of this review. The authors have no affiliation with any organization with a direct or indirect financial interest in the subject matter discussed in the manuscript.

\section{Author details}

${ }^{1}$ Institute of Exercise Training and Sport Informatics, German Sports University, Cologne, Germany. ${ }^{2}$ Institute of Physics, University of Freiburg, Freiburg im Breisgau, Germany. ${ }^{3}$ Bloomberg LP, New York, USA.

Received: 22 February 2021 Accepted: 9 October 2021

Published online: 30 October 2021

\section{References}

1. Harari YN. Homo Deus: a brief history of tomorrow. Homo Deus: Random House; 2016.

2. Rajšp A, Fister I. A systematic literature review of intelligent data analysis methods for smart sport training. Appl Sci. 2020;10:3013.
3. Roy R, Paul A, Bhimjyani P, Dey N, Ganguly D, Das AK, et al. A short review on applications of big data analytics. In: Mandal JK, Bhattacharya D, editors., et al., Emerg technol model graph. Singapore: Springer; 2020. p. 265-78.

4. Claudino JG, Cardoso Filho CA, Boullosa D, Lima-Alves A, Carrion GR, Gianonl RL da S, et al. The role of veracity on the load monitoring of professional soccer players: a systematic review in the face of the big data era. Appl Sci. 2021;11:6479.

5. Cottle M, Hoover W, Kanwal S, Kohn M, Strome T, Treister NW. Transforming health care through big data: strategies for leveraging big data in the health care industry. Inst. Heal. Technol. Transform. - iHT. 2013.

6. MacLennan T. Moneyball: The Art of Winning an Unfair Game. J Pop Cult. 2005;

7. Rein R, Memmert D. Big data and tactical analysis in elite soccer: future challenges and opportunities for sports science. Springerplus. 2016;5:1-13.

8. Raghupathi W. Data Mining in Health Care. [Internet]. 1st ed. Healthc. Informatics Improv. Effic. Product. Taylor \& Francis; 2010. https://www. taylorfrancis.com/books/e/9780429131059

9. Claudino JG, Capanema D de O, de Souza TV, Serrão JC, Machado Pereira AC, Nassis GP. Current approaches to the use of artificial intelligence for injury risk assessment and performance prediction in team sports: a systematic review. Sports Med Open Sports Med Open; 2019. p. 1-12.

10. Taborri J, Keogh J, Kos A, Santuz A, Umek A, Urbanczyk C, et al. Sport biomechanics applications using inertial, force, and EMG sensors: a literature overview. Appl Bionics Biomech. 2020;2020.

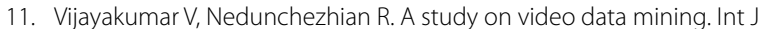
Multimed Inf Retr. 2012;1:153-72.

12. Bialkowski A, Lucey P, Carr P, Yue Y, Sridharan S, Matthews I. Large-scale analysis of soccer matches using spatiotemporal tracking data. In: Proceedings of the IEEE international conference on data mining, ICDM. 2015;2015-Janua:725-30.

13. Bunker RP, Thabtah F. A machine learning framework for sport result prediction. Appl Comput Inform. 2019;15:27-33. https://doi.org/10.1016/j. aci.2017.09.005

14. Colyer SL, Evans M, Cosker DP, Salo AIT. A review of the evolution of vision-based motion analysis and the integration of advanced computer vision methods towards developing a markerless system. Sport Med Open.; 2018;4. https://sportsmedicine-open.springeropen.com/ articles/https://doi.org/10.1186/s40798-018-0139-y

15. Raghupathi W, Raghupathi V. Big data analytics in healthcare: promise and potential. Heal Inf Sci Syst. 2014

16. Wang Y, Kung LA, Byrd TA. Big data analytics: understanding its capabilities and potential benefits for healthcare organizations. Technol Forecast Soc Change. 2018;126:3-13.

17. Yu KH, Beam AL, Kohane IS. Artificial intelligence in healthcare. Nat Biomed Eng. 2018;2:719-31.

18. Muzammal M, Talat R, Sodhro AH, Pirbhulal S. A multi-sensor data fusion enabled ensemble approach for medical data from body sensor networks. Inf Fusion. 2020;53:155-64.

19. Dong J, Zhuang D, Huang Y, Fu J. Advances in multi-sensor data fusion: algorithms and applications. Sensors. 2009;9:7771-84.

20. Gao W, Emaminejad S, Nyein HYY, Challa S, Chen K, Peck A, et al. Fully integrated wearable sensor arrays for multiplexed in situ perspiration analysis. Nature. 2016:529:509-14.

21. Mukhopadhyay SC. Wearable sensors for human activity monitoring: a review. IEEE Sens J. 2015;15:1321-30.

22. Kim J, Campbell AS, de Ávila BEF, Wang J. Wearable biosensors for healthcare monitoring. Nat Biotechnol. 2019;37:389-406. https://doi.org/10. 1038/s41587-019-0045-y.

23. Jeong IC, Bychkov D, Searson PC. Wearable devices for precision medicine and health state monitoring. IEEE Trans Biomed Eng IEEE. 2019;66:1242-58

24. Shi H, Zhao H, Liu Y, Gao W, Dou SC. Systematic analysis of a military wearable device based on a multi-level fusion framework: research directions. Sensors (Switzerland). 2019;19:2651.

25. Seshadri DR, Li RT, Voos JE, Rowbottom JR, Alfes CM, Zorman CA, et al. Wearable sensors for monitoring the physiological and biochemical profile of the athlete. NPJ Digit Med. 2019;2:1-16. https://doi.org/10.1038/ s41746-019-0150-9. 
26. Homayounfar SZ, Andrew TL. Wearable sensors for monitoring human motion: a review on mechanisms, materials, and challenges. SLAS Technol. 2020;25:9-24

27. Zhou H, Zhang Y, Qiu Y, Wu H, Qin W, Liao Y, et al. Stretchable piezoelectric energy harvesters and self-powered sensors for wearable and implantable devices. Biosens Bioelectron. 2020;168:112569. https://doi.org/10. 1016/j.bios.2020.112569.

28. Dinh T, Nguyen T, Phan HP, Nguyen NT, Dao DV, Bell J. Stretchable respiration sensors: Advanced designs and multifunctional platforms for wearable physiological monitoring. Biosens Bioelectron. 2020;166:112460. https://doi.org/10.1016/j.bios.2020.112460.

29. Heo JS, Eom J, Kim YH, Park SK. Recent progress of textile-based wearable electronics: a comprehensive review of materials, devices, and applications. Small. 2018;14:1-16.

30. Moran DS, Mendal L. Core temperature measurement: methods and current insights. Sport. Med. 2002.

31. Rice P, Upasham S, Jagannath B, Manuel R, Pali M, Prasad S. CortiWatch: watch-based cortisol tracker. Futur Sci OA. 2019;5:FSO416.

32. Wen W, Tomoi D, Yamakawa H, Hamasaki S, Takakusaki K, An Q, et al. Continuous estimation of stress using physiological signals during a car race. Psychology. 2017;6:978-86. https://www.researchgate.net/publi cation/317012834_Continuous_Estimation_of_Stress_Using_Physiologi cal_Signals_during_a_Car_Race

33. Chu M, Nguyen T, Pandey $\bar{V}$, Zhou Y, Pham HN, Bar-Yoseph $R$, et al. Respiration rate and volume measurements using wearable strain sensors. NPJ Digit Med. 2019;2:1-9. https://doi.org/10.1038/s41746-019-0083-3.

34. Imani S, Bandodkar AJ, Mohan AMV, Kumar R, Yu S, Wang J, et al. A wearable chemical-electrophysiological hybrid biosensing system for real-time health and fitness monitoring. Nat Commun. 2016;7:1-7.

35. Taelman J, Adriaensen T, Van Der Horst C, Linz T, Spaepen A. Textile integrated contactless EMG sensing for stress analysis. In: Annu Int Conf IEEE Eng Med Biol Proc. 2007. p. 3966-9.

36. Lin R, Kim HJ, Achavananthadith S, Kurt SA, Tan SCC, Yao H, et al. Wireless battery-free body sensor networks using near-field-enabled clothing. Nat Commun. 2020;11:1-10.

37. Johnston W, O'Reilly M, Argent R, Caulfield B. Reliability, validity and utility of inertial sensor systems for postural control assessment in sport science and medicine applications: a systematic review. Sport Med. 2019;49:783-818.

38. Malik A. RTLS for DUMMIES. Wiley Publ. 2009

39. Sengupta A, Jin F, Zhang R, Cao S. mm-Pose: real-time human skeletal posture estimation using mmWave radars and CNNs. IEEE Sens J. 2020;20:10032-44.

40. Mendoza-Silva GM, Torres-Sospedra J, Huerta J. A meta-review of indoor positioning systems. Sensors (Switzerland). 2019;19:4507.

41. De Silva V, Caine M, Skinner J, Dogan S, Kondoz A, Peter T, et al. Player tracking data analytics as a tool for physical performance management in football: a case study from chelsea football club academy. Sports. 2018:6:130.

42. Zhai C, Zou Z, Zhou Q, Mao J, Chen Q, Tenhunen H, et al. A 2.4-GHz ISM RF and UWB hybrid RFID real-time locating system for industrial enterprise Internet of Things. Enterp Inf Syst. 2017;11:909-26.

43. Kamel Boulos MN, Berry G. Real-time locating systems (RTLS) in healthcare: A condensed primer. Int. J. Health Geogr. 2012.

44. Clarinox. Real Time Location Systems. Clarinox.Com. 2009.

45. Ho HJ, Zhang ZX, Huang Z, Aung AH, Lim WY, Chow A. Use of a real-time locating system for contact tracing of health care workers during the COVID-19 pandemic at an infectious disease center in singapore: Validation study. J Med Internet Res. 2020;22.

46. Leser R, Baca A, Ogris G. Local positioning systems in (game) sports. Sensors. 2011;11:9778-97.

47. Khalid R, DAS GUPTA R, ALIZADEH P. Real-time location sensing system. 2018. https://patents.google.com/patent/WO2018206934A1/en

48. Wu T, Rappaport TS, Collins CM. The human body and millimeter-wave wireless communication systems: interactions and implications. IEEE Int Conf Commun. 2015. https://ieeexplore.ieee.org/document/7248688

49. Hsu CM, Shiu CW. 3D LiDAR-based precision vehicle localization with movable region constraints. Sensors (Switzerland). 2019;19.

50. Luo X, Wang H, Yan S, Liu J, Zhong Y, Lan R. Ultrasonic localization method based on receiver array optimization schemes. Int J Distrib Sens Netw.
2018;14. https://journals.sagepub.com/doi/full/https://doi.org/10.1177/ 1550147718812017

51. Pancham J, Millham R, Fong SJ. Investigation of obstructions and range limit on bluetooth low energy RSSI for the healthcare environment [Internet]. Lect. Notes Comput. Sci. (including Subser. Lect. Notes Artif. Intell. Lect. Notes Bioinformatics). Springer International Publishing; 2018. http://dx.doi.org/https://doi.org/10.1007/978-3-319-95171-3_21

52. Blauberger $P$, Marzilger $R$, Lames $M$. Validation of player and ball tracking with a local positioning system. 2021:21:3501-9

53. Thomas G, Gade R, Moeslund TB, Carr P, Hilton A. Computer vision for sports: current applications and research topics. Comput Vis Image Underst. 2017

54. Bastida Castillo A, Gómez Carmona CD, De la Cruz Sánchez E, Pino Ortega J. Accuracy, intra- and inter-unit reliability, and comparison between GPS and UWB-based position-tracking systems used for time-motion analyses in soccer. Eur J Sport Sci. 2018;18:450-7.

55. Dabove P, Di Pietra V. Towards high accuracy GNSS real-time positioning with smartphones. Adv Sports Res. 2019;63:94-102.

56. Movassaghi S, Abolhasan M, Lipman J, Smith D, Jamalipour A. Wireless body area networks: a survey. IEEE Commun Surv Tutorials. 2014;16:1658-86

57. Lacasa L, Nicosia V, Latora V. Network structure of multivariate time series. Sci Rep. 2015;5:1-9.

58. Jing L, Wang T, Zhao M, Wang P. An adaptive multi-sensor data fusion method based on deep convolutional neural networks for fault diagnosis of planetary gearbox. Sensors (Switzerland). 2017;17:414.

59. Kidziński Ł, Delp S, Schwartz M. Automatic real-time gait event detection in children using deep neural networks. PLOS ONE. 2019;14:1-11.

60. Malhotra P, Vishnu TV., Vig L, Agarwal P, Shroff G. TimeNet: Pre-trained deep recurrent neural network for time series classification. In: ESANN 2017-proceedings, 25th Eur Symp Artif Neural Networks, Comput Intell Mach Learn. 2017

61. Li J, Pedrycz W, Jamal I. Multivariate time series anomaly detection: a framework of Hidden Markov models. Appl Soft Comput J. 2017;60:22940. https://doi.org/10.1016/j.asoc.2017.06.035.

62. Kapp V, May MC, Lanza G, Wuest T. Pattern recognition in multivariate time series: towards an automated event detection method for smart manufacturing systems. J Manuf Mater Process. 2020;4:88.

63. Adaskevicius R. Method for recognition of the physical activity of human being using a wearable accelerometer. Elektron ir Elektrotechnika. 2014;20:127-31.

64. Shi $Y$, Shi $Y$, Wang $X$. Fall detection on mobile phones using features from a five-phase model. In: Proceedings of the- IEEE 9th international conference on Ubiquitous Intell Comput IEEE 9th Int Conf Auton Trust Comput UIC-ATC 2012. 2012;951-6

65. Vallejo M, Isaza C V., Lopez JD. Artificial neural networks as an alternative to traditional fall detection methods. In: Proceedings of the annual international conferene on IEEE Eng Med Biol Soc EMBS. 2013;1648-51

66. Mao Y, Qi H, Ping P, Li X. Contamination event detection with multivariate time-series data in agricultural water monitoring. Sensors (Switzerland). 2017;17:1-19.

67. Jimenez AM. Physiological sensor. ProQuest Diss Theses. 2013;139. http://search.proquest.com/docview/1527176270?accountid $=6180 \%$ 5Cnhttp://dw2zn6fm9z.search.serialssolutions.com/?ctx_ver=Z39.882004\&ctx_enc=info:ofi/enc:UTF-8\&rfr_id=info:sid/ProQuest+Dissertatio ns+\%26+Theses+Global\&rft_val_fmt=info:ofi/fmt:kev:mtx:dissert

68. Mursalin M, Zhang Y, Chen Y, Chawla NV. Automated epileptic seizure detection using improved correlation-based feature selection with random forest classifier. Neurocomputing. 2017;241:204-14.

69. Porciuncula F, Roto AV, Kumar D, Davis I, Roy S, Walsh CJ, et al. Wearable movement sensors for rehabilitation: a focused review of technological and clinical advances. PM R. American Academy of Physical Medicine and Rehabilitation; 2018;10:S220-32. https://doi.org/10.1016/j.pmrj.2018.06. 013

70. Shenavar Masooleh I, Zayeni H, Haji-Abbasi A, Azarpira M, Hadian A, Hassankhani A, et al. Cardiac involvement in rheumatoid arthritis: a crosssectional study in Iran. Indian Heart J. 2016.

71. Ždárská D, Pelíšková P, Charvát J, Slavíček J, Mlček M, Medová E, et al. ECG body surface mapping (BSM) in type 1 diabetic patients. Physiol Res. 2007;56:403-10. 
72. Abela M, Sharma S. Abnormal ECG findings in athletes: clinical evaluation and considerations. Curr Treat Options Cardiovasc Med. 2019;21:1-17.

73. Gholamhosseini L, Sadoughi F, Safaei A. Hospital real-time location system (A practical approach in healthcare): a narrative review article. Iran J Public Health. 2019;48:593-602.

74. Low B, Coutinho D, Gonçalves B, Rein R, Memmert D, Sampaio J. A systematic review of collective tactical behaviours in football using positional data. Sport. Med. 2020.

75. Papi E, Osei-Kuffour D, Chen YMA, McGregor AH. Use of wearable technology for performance assessment: a validation study. Med Eng Phys. 2015;37:698-704. https://doi.org/10.1016/j.medengphy.2015.03.017.

76. Kobsar D, Osis ST, Boyd JE, Hettinga BA, Ferber R. Wearable sensors to predict improvement following an exercise intervention in patients with knee osteoarthritis. J Neuroeng Rehabil. 2017;14:1-10.

77. Chambers R, Gabbett TJ, Cole MH, Beard A. The use of wearable microsensors to quantify sport-specific movements. Sport Med. 2015:45:1065-81.

78. O'Reilly M, Caulfield B, Ward T, Johnston W, Doherty C. Wearable inertial sensor systems for lower limb exercise detection and evaluation: a systematic review. Sport Med. 2018;48:1221-46.

79. James N. Notational analysis in soccer: past, present and future. Int J Perform Anal Sport. 2006;6:67-81. https://doi.org/10.1080/24748668.2006. 11868373.

80. Ali S, Khusro S, Rauf A, Mahfooz S. Sensors and mobile phones: evolution and state-of-the-art. Pak J Sci. 2014;66:386-400.

81. Gupta A, Chakraborty C, Gupta B. Medical information processing using smartphone under loT framework. Energy Conserv; 2019. https://doi.org/ 10.1007/978-981-13-7399-2 12

82. Lima WS, Souto E, El-Khatib K, Jalali R, Gama J. Human activity recognition using inertial sensors in a smartphone: an overview. Sensors (Switzerland). 2019

83. Vargason T, Howsmon DP, McGuinness DL, Hahn J. On the use of multivariate methods for analysis of data from biological networks. Processes. 2017;5:36.

84. Katz MH. Multivariable analysis: a practical guide for clinicians and public health researchers. Multivariable Anal A Pract. Guid. Clin. Public Heal. Res. 2011. https://www.cambridge.org/core/books/multivariable-analysis/ DBE7816A781AEF53108FD721199B4AC9

85. Reid RC, Mahbub I. Wearable self-powered biosensors. Curr Opin Electrochem. 2020;19:55-62.
86. Khoshmanesh F, Thurgood P, Pirogova E, Nahavandi S, Baratchi S. Wearable sensors: at the frontier of personalised health monitoring, smart prosthetics and assistive technologies. Biosens Bioelectron. 2021;176:112946.

87. Hajar R. The pulse from ancient to modern medicine: Part 3. Hear Views. 2018;19:117-20. https://www.ncbi.nlm.nih.gov/pmc/articles/PMC64 48473/

88. Duraisamy R, Dinakar S, Venkittaramanujam $\mathrm{V}$, Jeyakumar $\mathrm{V}$. A systematic approach for pulse diagnosis based on siddha medical procedures. In: 2017 4th Int Conf Signal Process Commun Networking, ICSCN 2017. 2017. https://ieeexplore.ieee.org/document/8085694

89. Zhang J, Niu X, Yang XZ, Zhu QW, Li HY, Wang X, et al. Design and application of pulse information acquisition and analysis system with dynamic recognition in traditional Chinese medicine. Afr Health Sci. 2014:14:743-52.

90. Lan KC, Litscher G, Hung TH. Traditional chinese medicine pulse diagnosis on a smartphone using skin impedance at acupoints: a feasibility study. Sensors (Switzerland). 2020;20:1-14.

91. Silver D, Hubert T, Schrittwieser J, Antonoglou I, Lai M, Guez A, et al. A general reinforcement learning algorithm that masters chess, shogi, and Go through self-play. Science (80- ). 2018:362:1140-4.

92. Crosby V, Wireless G. Body area networks for healthcare: a survey. Int J Ad hoc Sens Ubiquitous Comput. 2012;3:1-26.

93. Mathur A, Gupta CP. Big data challenges and issues: a review. Lect. Notes Data Eng. Commun. Technol. Springer; 2020. https://doi.org/10.1007/ 978-3-030-24643-3_53

94. Kluge EHW. Artificial intelligence in healthcare: ethical considerations. Healthc Manag Forum. 2020;33:47-9.

95. Gómez-González E, Gomez E, Márquez-Rivas J, Guerrero-Claro M, Fernández-Lizaranzu I, Relimpio-López MI, et al. Artificial intelligence in medicine and healthcare: a review and classification of current and nearfuture applications and their ethical and social Impact. 2020. http://arxiv. org/abs/2001.09778

\section{Publisher's Note}

Springer Nature remains neutral with regard to jurisdictional claims in published maps and institutional affiliations.

\section{Submit your manuscript to a SpringerOpen ${ }^{\circ}$ journal and benefit from:}

- Convenient online submission

- Rigorous peer review

- Open access: articles freely available online

- High visibility within the field

- Retaining the copyright to your article

Submit your next manuscript at springeropen.com 